\title{
3D ANTHROPOMETRY IN ERGONOMIC PRODUCT DESIGN EDUCATION
}

\author{
Anton JELLEMA ${ }^{1}$, Evan GALLOUIN² ${ }^{2}$ Benoît MASSÉ ${ }^{2}$, lemkje RUITER ${ }^{1}$, Johan \\ MOLENBROEK ${ }^{1}$ and Toon HUYSMANS ${ }^{1}$ \\ ${ }^{1}$ University of Technology, Faculty IDE, Delft $2628 \mathrm{CE}$, The Netherlands \\ ${ }^{2}$ Student ESTIA Institute of Technology, 64210 Bidart - France
}

\begin{abstract}
The Faculty of Industrial Design Engineering of the Delft University of Technology offers a bachelor's degree education programme and three master's programmes. Our students are lectured in ergonomics and learn to design and conduct research in ergonomics. In this paper we describe the development of methods to realise ergonomic fit mapping based on 3D anthropometrics and to educate students on this topic.

Due to the increasing availability of 3D scan data, we enter the complex field of 3D anthropometry and statistical shape models, which is an increasingly popular mathematical representation for 3D human shape variation. These facilities and knowledge are particularly useful when it comes to products that should fit close to the human body.

The use of 3D anthropometrics is explained and practiced throughout the different stages of complexity. It starts with the use of 1D and 2D anthropometric data, the application of percentiles and the DINED tool Ellipse to see the correlation between two different body dimensions and to determine the consequences for related product dimensions. It ends with the use of 3D anthropometric data for the design of a helmet for cyclists, by way of bi-variate based shape analysis of the head. We made efforts to lower the burden for students working with 3D scan data, for example by providing pre-processed 3D scan databases and casus specific measurement tables.
\end{abstract}

Keywords: Design education, 3D human shape variation, ergonomic fit mapping

\section{INTRODUCTION}

Sizing systems for products that need to fit close to the body - like helmets, EEG headsets and snorkelling masks - generally use the variation of one or two body dimensions to be directly applied to the product. But this information rarely covers the complexity of the human form [1]. The multivariate anthropometrics capture most of the variation of the human shape with a limited number of variables. The most common approach consists in conducting a principal component analysis (PCA) on the dataset. However, as the principle components have no clear physical interpretation in terms of product dimensions (for example circumference or width) more intuitive parameters, directly related to the future product are needed to design it [2] [3]. Although many researches show that this is possible [4], there are still no tools available for designers to help them incorporate this knowledge in their design process. The ultimate goal would be to implement this knowledge by using a statistical or anthropometric shape model of the target group in CAD programs [5]. Other ways to implement 3D anthropometrics in the design process are $3 \mathrm{D}$ printing of prototypes or body parts to evaluate the ergonomic fit. The 3D anthropometric approach also has potential for attaining a personalised product fit, e.g. with 3D scanning and additive manufacturing, where it can be employed for 3D scan interpretation and design automation [6]. This paper describes the approach adopted by the Faculty of Industrial Design Engineering (IDE) of the Delft University of Technology to educate our students in this field and tries to challenge the CAD software industry.

\section{ANTHROPOMETRY AND HUMAN CENTRED DESIGN}

\subsection{Anthropometric data: collectors and users}


There are two groups of users involved when it comes to anthropometric data. On the one hand, we have the researchers that collect anthropometric data, which might also be users of the data and, on the other hand, we have the designers who analyse the data to extract relevant insights to steer their product design, but who are usually not involved in the collection of the data. At the TU Delft we try to bridge the gap between these two groups. Our goal is to present collected data in a designer (user) friendly way. To achieve this, we involve our students in the process of developing the data presentation. In the past, we looked at the use of 1D and 2D data and 3D digital human models. In order to make sure that our students will be able to exploit the opportunities provided with the developments in 3D scanning, we extend our focus to the presentation and use of 3D scan data even though the way to present the data can be seen as work in progress. This will also enlarge our insight into how designers use shape information of the human body. This approach is supported by Luximon et al [7].

\subsection{Anthropometry in the human centred design process}

The development process of products could be divided into three stages: analysis, conceptualisation and embodiment [8]. The use of anthropometric data during the design process shows similar stages. In the analysis stage, the designer defines the intended user group for the product and which body and product measurements are relevant in the human-product-interaction. Who are the users? What demographic background do they have, what is their age and gender? Is the product intended for a group of users or for a single user? Will the product come in a variety of sizes, be adaptable or be a 'one-size-fits-all'? The designer needs also insight in what body positions the users might have while using the product or what body positions the product needs to support, how close the product will get to the human body, and the forces which are at play. Doing observations is an important way to get insight in all these aspects.

Based upon the outcome of the exploration a choice can be made for the appropriate anthropometric database to use in the concept stage. In this phase, usually 1D databases will be suitable to determine the outlines of the product dimensions. For certain products, the designer wants to know the correlation between two anthropometric sizes, also called 2D anthropometry. For instance, the dimensions of a chair can depend on how the lower leg length is correlated to the upper leg length. In addition, 3D digital human models can be very useful in this stage to assess the dimensions of the product or accommodation - like a cabin - in relation to the postures and movements of the user.

In the embodiment stage, the exact dimensions of the product need to be determined. The types of databases and tools to achieve this will depend on the type of product. When there is no contact between user and product, 1D anthropometric data will usually be sufficient. The larger the part of the body coming in close contact with the product, the more likely information about the body shape will be needed. In that case, using 3D scan data will be necessary to develop a proper fitting product.

\section{ERGONOMIC PRODUCT DESIGN EDUCATION}

\subsection{Learning objectives}

The education in anthropometry, as part of the education at the Faculty IDE, has several objectives.

The first goal is to provide the students with knowledge about existing anthropometric databases. They should get familiar with 1D, 2D, and 3D anthropometric databases and have insight into how the data for these databases have been collected. The next goal is to learn how to select the most appropriate anthropometric database. Finally, the students should be able to apply the anthropometric data in the product design process, e.g. selecting critical product and body dimensions and devising sizing systems or adjustable product parts. This data is accessible via the DINED platform [9] and the faculty has 3D scanning facilities available (several Artec hand scanners, access to Artec software and a full body scanner (3DMD), including 1FTE dedicated technical staff and measurement support, as well as support in 3D anthropometric data analysis by a staff member). Another learning objective is to educate our students in the use of digital design software and to keep up with the technological developments of these more and more complex systems [10].

To achieve these learning objectives, we have set up a programme of education in anthropometry that makes use of the stages of the design process. In the bachelor's course, students learn the basics and they expand their knowledge in the master's course. 


\subsection{Anthropometry in the bachelor of the Faculty IDE}

The anthropometry course in the first year of the bachelor consists of a combination of lectures (in the morning) with corresponding group assignments (4 to 5 students per group, more than 300 students in total) in the afternoon. As our students have no prior knowledge of anthropometry, we start with 1D anthropometry. We explain how measurements of the human body are taken and have the students measure some body dimensions themselves. They have to compare their own dimensions with the collected data of the Dutch population as well as with the data collected from Dutch students, as available from our DINED platform [9].

In the course, we use a non-adjustable bicycle helmet to demonstrate the use and usefulness of anthropometric data in the design process. When one buys a helmet, the required dimension to select the right size of helmet is one's head circumference. Therefore, students are asked to measure their own head circumference as well as head width and head depth. The helmet we use only comes in sizes M and L. The students have to choose the helmet that should fit their head (based on their head circumference) and give a review of their experience with the fit of the helmet.

The second step is teaching the students how to deal with correlation of body measurements. They are introduced to the interactive Ellipse program on our DINED platform. The students have to enter the head width, depth, and circumference data of the Dutch population in Ellipse to determine the width and depth of two sizes of bicycle helmets. These helmets should fit the widest possible part of the population. At this point the students have determined the width and depth of the helmets, but not yet the shape. To find out the optimal shape of the helmets, they can use a spreadsheet with digital and virtual head measurements extracted from a database of 3D body scans [11]. First of all, they have to select those individuals that have a depth and width that is smaller than the depth and width they chose for their helmets. A set of contours extracted from the same database is also provided. See Figure 1 for explanation of the contour line. They get a step-by-step procedure on how to display these contours in Paraview [12]. Based on a visualisation of all the selected cross sections in one picture, the students draw the optimal contour for the helmets. See Figure 1.
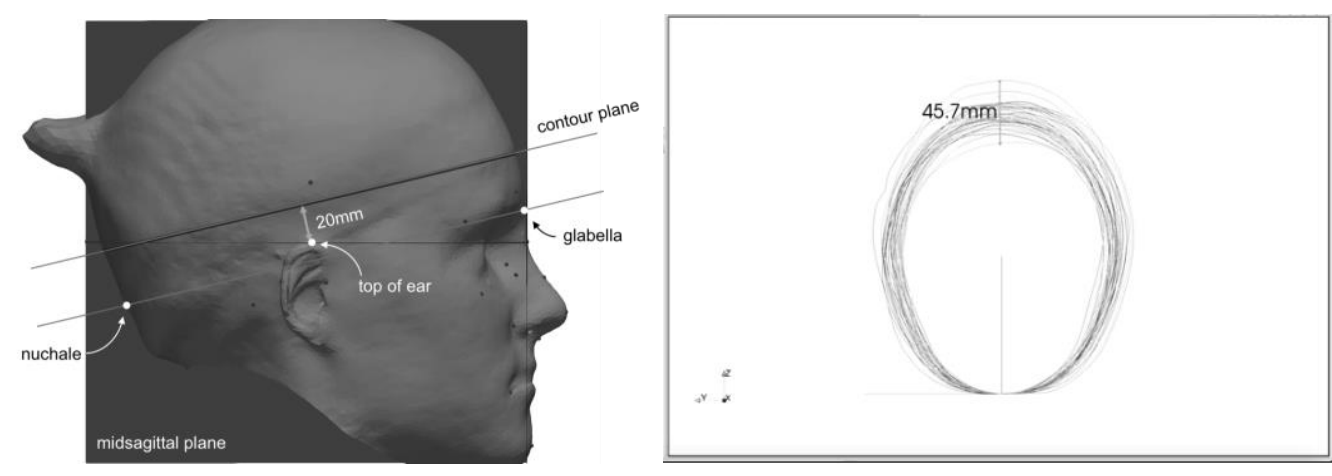

Figure 1. Left: sideview 3D scan (head with hairnet) for definition of the location and orientation of the head contour. Right: simultaneous visualisation of several head contours in Paraview

At this point the students are also able to determine the thickness of the foam layer inside the helmet that should help accommodate different head shapes within one helmet size. One student (Lukas Bannink) was even able to present the helmet his team designed in Blender [13]. See Figure 2.

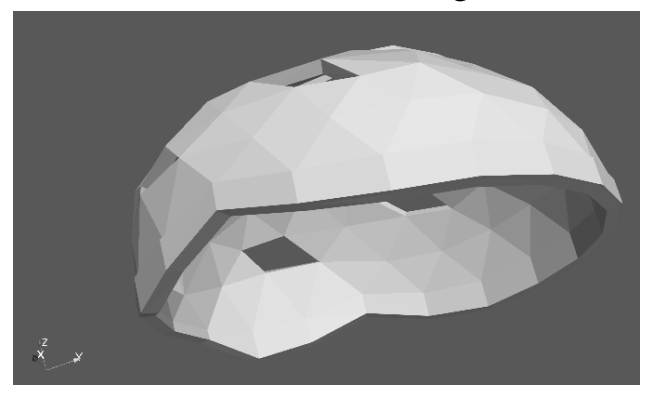

Figure 2. Helmet design of one team in Blender 


\subsection{Anthropometry in the master's Integrated Product Design (IPD)}

At IDE we have three master's education programmes, each with about 100-120 students. One of these programmes is the IPD master's, with two courses where ergonomics and anthropometrics are important: Advanced Concept Design and Advanced Embodiment Design. Part of these courses is coaching during the projects by experts in ergonomics and a 3D scan symposium including a 3D scan workshop.

The master's elective 'Biomechanics and Digital Human Modelling' gives an in-depth treatment of modelling and simulation techniques. The course focuses on musculoskeletal biomechanics and 3D anthropometrics. For the part focusing on 3D anthropometry, students are introduced to various 3D scanning techniques for data collection. Also, they extend their knowledge to multivariate anthropometric analyses. Multivariate techniques, like PCA, allow them to treat their data in a more comprehensive way, generating new insights for product improvement. Further, the students are taught multivariate treatment of measurement data and 3D scanned surface data, also termed 3D surface anthropometry. As a background, an overview is given on the state of the art of surface correspondence methods which are employed to prepare 3D scan data for further statistical analysis. This is followed by introducing the concept of statistical shape models which, by means of PCA, model the shape variation present in a 3D scan database (i.e. population sample) with a compact set of parameters. See Figure 3. Finally, they are shown how these anthropometric shape models can be used to generate 3D design manikins controlled by intuitive parameters (e.g. length, weight), which can be employed in the design process. The theory is concluded with an overview of applications of 3D anthropometry, ranging from sizing system development for garments, helmets and headsets, design automation for ultra-personalised products (like wrist splints and spectacle frames), to virtual fit mapping of orthopaedic implants.

The learned concepts of statistical shape models are demonstrated by the students in a workshop and brought into practice via a design challenge around a specific product, for example a sports brace to avoid ankle sprains. For the anthropometric part, the students are provided with an ankle data set consisting of 3D scans and measurements of the ankle of 900 subjects derived from the CAESAR database [11], already prepared for statistical analysis (i.e. brought into correspondence). With this data they can create 3D design manikins in Paraview. The students can incorporate these manikins into their design.

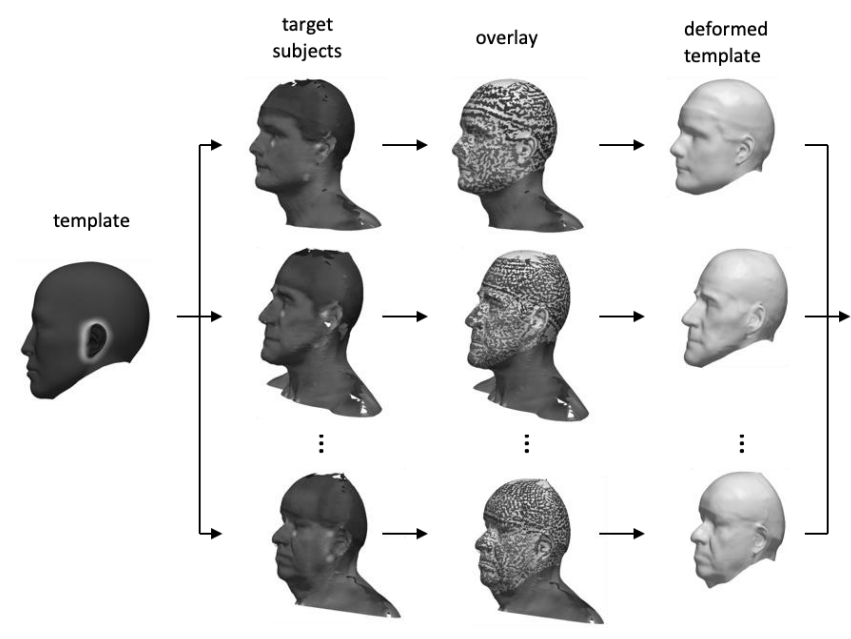

correspondence process

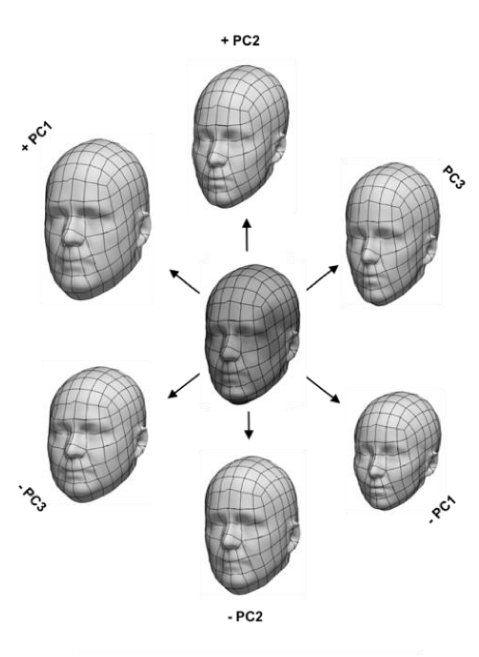

shape model

Figure 3. Schematic overview of the process of building a statistical shape model from a collection of $3 D$ scans. From left to right: a template surface is registered to the $3 D$ scan of each subject in the collection, resulting in a representation of the different body shapes with

a consistent mesh, i.e. an equal number of points and at corresponding anatomical locations. From the consistent meshes a shape model is derived by the use of principal components analysis. The model captures the main variance in the data set with a limited number of variables, termed shape modes. A visualisation of first three shape modes (PC1,

$P C 2, P C 3)$ is shown at the right: the mean the shape is at the centre and the extreme shapes corresponding to an offset of $+/$ - three standard deviations along the principal components, are shown at the end of the diagonals 


\subsection{Summer internship}

Because we were curious to find out what the possibilities are for ergonomic fit evaluation with Artec studio, we asked students from ESTIA Institute of Technology, France, who come for a summer internship at our faculty, to carry out an evaluation. The steps of this method will be described here, based on a test with the Decathlon Easybreath (small and large size) mask [14].

It is important to place the scanned Easybreath mask on the 3D scan of the face in such a way that the product is placed in a natural way. First, a copy of the real-life situation is made: a 3D scan of the face with the Easybreath mask on. Then, the mask itself is scanned as well as the face without the product on. With the help of markers on the face, the "face-only" is aligned to the "face-with-product". Next, the scan of the "product-only" is aligned and the face with the product is deleted, resulting in the product virtually placed on the face in a natural way. In the next phase, they produced four average digital head forms based on the bi-variate distribution of head circumference and face length. Now the distances between these four head forms and the mask can be measured as the fit mapping was done with Artec Studio. To compare the outcomes, they also 3D milled a foam head form to physically evaluate the mask fit. See figure 4.

Besides the effort described above, to place the mask in a natural way on the face, the designer also needs to have a proper definition of fit. The Easybreath mask needs to fit watertight, the glasses and mask need to be comfortable, which means that the straps cannot be too tight for this would cause too much pressure on specific places of the head. Anatomical knowledge is also important to know what places of the head can bear some pressure and what places cannot (arteries, nerves, bone, skin).
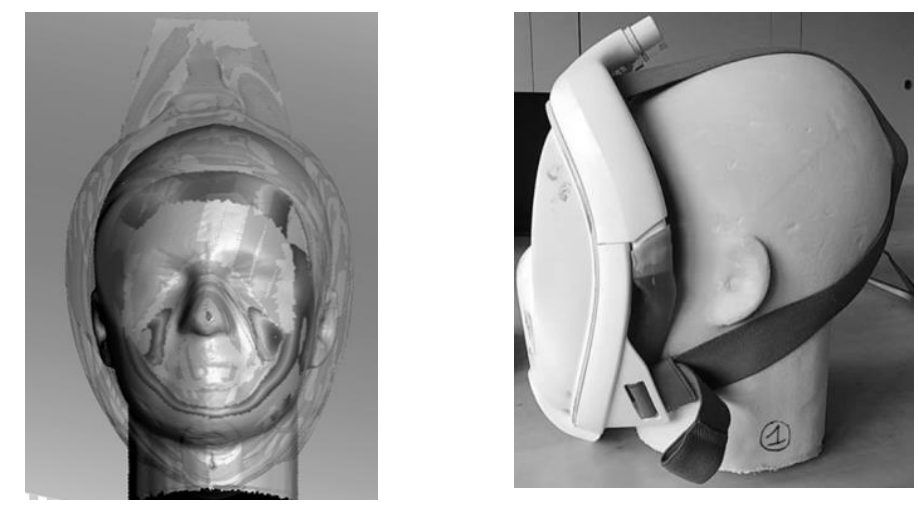

Figure 4. Left: heatmap of the distances between one of the four head forms (the smallest one) and the small mask in Artec studio. Heatmap: red means a gap, blue means that the flexible part of the mask is pushed close to the face. Right: fit test with real mask and head form

\section{Discussion}

This paper described the way we introduce the use of 3D scan data to our Industrial Design Engineering students. First thing we learned is that it is very important that students have some knowledge about the way data are collected. Second, they need a basic understanding of the relation between body measurements and product dimensions. Building this up in the bachelor course from 1D and 2D to 3D did help, as well as the choice of product (a helmet, which is a close to the body product, and not too complicated because the head is quite solid). However, even though we prepared a very limited database for them (with preselected contours), it took them some time to grasp the method of how to work with these contours. In this stage they would not have been able to produce the contours from the database themselves. In the master's, doing the scanning of the data themselves helped the students to get more insight into the gathering of scan data. Because they have gained experience in dimensioning products during their bachelor, they are better able to understand the human-product interaction and the dimensions that are important. They also have a better understanding of the variation in body dimensions. But the statistical shape models also need to be prepared for them. This proved that it is very important to tailor the presentation of the scan data to the level of knowledge of the students. 
When it comes to virtual fit mapping with the Easybreath mask, we learned that the main problem was defining the fit. Fit on the face involves fit on both hard and soft tissue and this relates also to the flexibility of the contact surface of the product. Thus, you do need background knowledge of the relevant positions on the face, but also of the anatomical characteristics before you are able to predict fit. User testing with a prototype is necessary. Validation of the data and methods is needed.

\section{CONCLUSIONS}

Concluding, the most important challenge is tailoring the presentation of $3 \mathrm{D}$ anthropometric data to the level of knowledge of our students and designers. The focus of our research is not to bring up the level of knowledge of bachelor students to a specialist level, but to incorporate this specialist knowledge into the presentation of the data. Our DINED platform is built around a growing number of data sets of 1D measurements and, through its interactive interface, it provides a user friendly uni- and bivariate anthropometry tool for designers. We are currently extending this platform to include 3D surface anthropometry tools and 3D surface data sets in order to disseminate our knowledge to our bachelor students and the industry. The summer internships give the possibility to experiment with new tools, before we introduce these tools in our educational programme. Our master's students have the opportunity to deepen the knowledge or gather new data in electives or their graduation project.

\section{ACKNOWLEDGEMENT}

Lukas Bannink ( $1^{\text {st }}$ year student bachelor Industrial Design) for his helmet design.

\section{REFERENCES}

[1] Goto L., Huysmans T., Lee W., Molenbroek J.F. and Goossens R.H.M. "A Comparison Between Representative 3D Faces Based on Bi-and Multi-variate and Shape Based Analysis.," in Congress of the International Ergonomics Association, 2018.

[2] Lacko D., Huysmans T., Parizel P.M., De Bruyne G., Verwulgen S., Van Hulle M.M. and Sijbers J. "Evaluation of an anthropometric shape model of the human scalp," Applied Ergonomics, vol. 48, pp. 70-85, 2015.

[3] Lacko D. "The application of 3D anthropometry for the development of headgear-a case study on the design of ergonomic brain-computer interfaces.," Antwerpen, Belgium: Antwerpen, 2017.

[4] Danckaers F., Huysmans T., Lacko D. and Sijbers J. "Evaluation of 3D body shape predictions based on features.," in Proceedings of the 6th International Conference on 3D Body Scanning Technologies, Lugano, Switzerland, 2015.

[5] Huysmans T., Danckaers F., Vleugels J., Lacko D., De Bruyne G., Verwulgen S. and Sijbers J. "Multi-patch B-spline statistical shape models for CAD-compatible digital human modelling.," in International Conference on Applied Human Factors and Ergonomics (AHFE), 2018.

[6] Verwulgen S., Lacko D., Vleugels J., Vaes K., Danckaers F., De Bruyne G. and Huysmans T. "A new data structure and workflow for using 3D anthropometry in the design of wearable products," International Journal of Industrial Ergonomics, vol. 64, pp. 108-117, 2018.

[7] Luximon Y., Luximon A. and Fu F. "Rethinking Ergonomics in Design," in International Conference on Applied Human Factors and Ergonomics (AHFE), 2018.

[8] Van Boeijen A., Daalhuizen J., van der Schoor R. and Zijlstra J. The Delft design guide: Design strategies and methods, Delft, 2014.

[9] Molenbroek J. "DINED: Anthropometry in Design," TU Delft, [Online]. Available: www.dined.nl.

[10] Aldoy N. and Evans M. "A review of digital industrial and product design methods in UK higher education," The Design Journal, vol. 14, no. 3, pp. 343-368, 2011.

[11] Robinette K. M., Blackwell S., Daanen H., Boehmer M. and S. Fleming, "Civilian American and European Surface Anthropometry Resource (CAESAR), Final Report. Volume 1. Summary," SYTRONICS INC DAYTON OH, 2002.

[12] Ayachit U. "The ParaView Guide: A Parallel Visualisation Application," Kitware, 2015.

[13] Blender, "Open source 3D creation," [Online]. Available: https://www.blender.org. [Accessed 11 March 2019].

[14] "Subsea Easybreath surface snorkelling mask," Decathlon, [Online]. Available: https://www.decathlon.co.uk/easybreath-mask-turquoise-id_8401951.html. [Accessed 10 March 2019]. 